MAX-EMANUEL GEIS

Friedrich-Alexander-Universität Erlangen-Nürnberg max-emanuel.geis@fau.de

KEVIN FRANK

Friedrich-Alexander-Universität Erlangen-Nürnberg kevin.frank@fau.de

\title{
Raumplanung in Deutschland und Polen - eine rechtsvergleichende Skizze
}

\begin{abstract}
Zusammenfassung: Die Geschichte der Raumplanung in Deutschland und Polen geht weit zurück. Dabei bestehen neben einzelner raumplanerischer auch gemeinsame und europäische Ansätze für die Raumnutzung. Der Aufsatz zeigt zunächst den rechtlichen Rahmen auf nationaler und supranationaler Ebene auf. Hiernach erfolgt eine vertiefende Darstellung anhand mehrerer Projekte aus dem Bereich der Energiewirtschaft und der Infrastruktur. Abschließend werden die Gemeinsamkeiten und Unterschiede herausgearbeitet.
\end{abstract}

Schlüsselwörter: Gemeinsamkeiten, Offshore-Windparks, Atomkraftwerk, Autostrada A1, Zentralflughafen.

\section{Einführung}

To understand a science it is necessary to know its history.

Auguste Comte

Raumplanung ist seit geraumer Zeit ein wichtiges Spielfeld des Europarechts. Dass sie schon aufgrund ihres dimensionalen Zugriffs nicht an Staatsgrenzen halt machen kann und darf, ist spätestens seit den 80er Jahren bekannt. ${ }^{1}$ In der

* Max-Emanuel Geis ist Inhaber des Lehrstuhls für Deutsches und Bayerisches Staats- und Verwaltungsrecht ans der Friedrich-Alexander-Universität Erlangen-Nürnburg, Kevin Frank ist wissenschaftlicher Mitarbeiter an diesem Lehrstuhl. 
Folge hat das Europarecht das Erfordernis zu einer grenzüberschreitenden Abstimmung zwischen den Mitgliedstaaten immer weiter konkretisiert. ${ }^{2}$ Dem 1993 von der Kommission vorgelegten Konzept „Europa 2000“ folgte 1999 das Europäische Raumentwicklungskonzept (EUREK) sowie 2007 die Territoriale Agenda der Europäischen Union (TAEU). ${ }^{3}$ Im Vertrag von Lissabon fand diese Entwicklung mit dem Konzept der territorialen Kohäsion ihren vorläufigen Abschluss, das auch in den AEUV integriert wurde. Eine wesentliche Initialwirkung geht bei dieser Entwicklung sicher von der sog. „SUP-Richtlinie“ aus, die die zwischenstaatliche Auswirkung von Planungsmaßnahmen erstmals zentral in den Fokus nahm. ${ }^{4}$

Die Auswirkungen hoheitlicher Entscheidungen auf benachbarte Herrschaftsgebilde hat nun freilich eine 1000-jährige Tradition: Bereits im 10. Jahrhundert leitete der ostfränkische König Heinrich I., u.a. zum Schutz vor einfallenden Stämmen, einen großangelegten Burgenbau auf der rechtlichen Grundlage einer Burgenordnung auf deutschem Boden ein. ${ }^{5}$ Doch muss diese Herangehensweise deutlich fokussiert werden. Darum springen wir einige Jahrhunderte nach vorne und betrachten - im Hinblick auf die seit Ende der 1940er etablierten sozialen Marktwirtschaft ${ }^{6}$ - die enorme Planungs- und Baudynamik in den 1970er Jahren, die v.a. durch die sozialliberale Politik unter Willy Brandt vorangetrieben wurde. ${ }^{7}$ Dieser Phase vorangegangen, war das unter Heinrich Lübke verkündete Raumordnungsgesetz $\left(\mathrm{ROG}^{8}\right)$ von 1965. ${ }^{9}$ Vor allem die Sorge vor einer schier unaufhaltsamen Versiegelungstätigkeit führte dazu, dass 1971 das Städtebauförderungsgesetz auf den Weg gebracht wurde, ${ }^{10}$ das u.a. den Zweck hatte, einer raschen Verstädterung durch gezielte Planung und Raumordnung entgegenzuwirken. ${ }^{11}$ Insoweit war die sozialliberale Politik Brandts sehr greifbar, die Modernisierung

${ }^{1}$ Vgl. U. Battis, J. Kersten, Europäische Raumentwicklung, „EuR“ 1, 2009, S. 3; D. Dörr, Raumordnung und Landesplanung, [in:] Bes. VwR, hrsg. D. Ehlers, M. Fehling, H. Pünder, Bd. 2, Heidelberg 2020, § 38, Rdn. 74 .

${ }^{2}$ Dazu R. Steenblock, I. Hartwig, Die Agenda 2007: Solidarität, Nachhaltigkeit und Innovation, ,integration“ 27, 2004, S. 85 ff.: Das Programm wird weitergeführt durch die TAEU 2020 und TAEU 2030.

${ }^{3}$ Vgl. dazu etwa C. Langhagen-Rohrbach, Raumordnung und Raumplanung, Darmstadt 2010, S. 17; D. Dörr, op. cit., S. 35.

${ }^{4}$ SUP-Richtlinie von 27.6.2001 (2001/42/EG), Art. 7; dazu etwa J. Schumacher, Umweltprüfung, [in:] Raumordnungsgesetz, hrsg. K. Werk, J. Albrecht, Wiesbaden 2012, § 9 Rdn. 1-3.

${ }^{5}$ G.U. Großmann, Die Welt der Burgen, München 2013, S. 107.

${ }^{6}$ R. Ptak, Vom Ordoliberalismus zur Sozialen Marktwirtschaft, Wiesbaden 2004, S. 9.

${ }^{7}$ H. Grebing, G. Schöllgen, Willy Brandt, Bonn 2013, S. 16, 52.

${ }^{8}$ Vgl. BGBl. 2020 I, S. 1328 (1347): Letzte Änderung des dieses Gesetzes durch Art. 159 VO (am 19. Juni 2020).

${ }^{9}$ BGBl. [1965] I S. 306.

${ }^{10}$ BGB1. [1971] I S. 1125.

${ }^{11}$ H. Grebing, G. Schöllgen, op. cit., S. 52. 
alter Gebäude ${ }^{12}$ und die Schaffung neuer Ortschaften, ${ }^{13}$ die nach Errichtung zweifelsfrei der breiten Bevölkerung in Form von neuem, bezahlbarem Wohnraum zugutekommen solle, zu forcieren. Nach der Wiedervereinigung Deutschlands im Oktober 1990 kam neben dem Versuch die wohnungstechnischen Unterschiede zwischen Ost und West auszugleichen, eine relativ hohe Geburtenrate in der BRD, die zu dieser Zeit seit Ende der 1980er konstant anstieg. ${ }^{14}$ Um dieser Aufgabe Herr zu werden, entwickelte man das Wohnungsbau-Erleichterungsgesetz (WoBauErlG) ${ }^{15}$ welches v.a. Genehmigungsverfahren beschleunigte.

Auf polnischer Seite entwickelte sich das Planungsrecht auf ganz andere Art und Weise. Nach dem Ende des zweiten Weltkriegs war Polen durch die Zugehörigkeit zum Warschauer Pakt mehr und mehr in die sowjetische Einflusssphäre geraten, was einerseits zu immensen gesellschaftlichen Spannungen führte ${ }^{16}$ und andererseits die Planungstätigkeit dem kommunistischen Staatsapparat unterwarf. ${ }^{17}$ Die kommunistischen Strukturen waren im ökonomischen Sinne auf eine geplante, vom Staat - im Blick auf Qualität und Stückzahl - vorgegebene Wirtschaft ausgerichtet. ${ }^{18}$ Spielräume für ein gewisses $\mathrm{Ma}$ an sinnvoller Verwirklichung waren nicht vorgesehen. ${ }^{19} \mathrm{Im}$ wirtschaftlichen Mittelpunkt stand das sozialistische Eigentum, ${ }^{20}$ dessen Entwicklung - vor allem durch die Planhoheit der Staatsorgane - gegenüber allen anderen, nicht-staatlichen Einflüssen verborgen blieb; es handelte sich nicht um eine gänzlich öffentliche Sache. ${ }^{21}$ Durch derartige kommunis-

$12 \S 21$ I StBauFG [Fassung: 1971].

13 § 1 III Nr. 1 [Fassung: 1971].

14 Statistisches Bundesamt, 11. Koordinierte Bevölkerungsvorberechnung, Bericht, 2006, S. 9 (Schaubild: signifikanter Anstieg von 1985-1990 im Westen), https://www.destatis.de/DE/Themen/ Gesellschaft-Umwelt/Bevoelkerung/Bevoelkerungsvorausberechnung/Publikationen/DownloadsVorausberechnung/bevoelkerung-deutschland-2050-bericht-5124203069004.pdf?_blob=publicationFile (abgerufen: 27.03.2021).

15 BGBl. [1990] I S. 962: Obwohl dieses auf 5 Jahre Bestand konzipiert war, wurde das Gesetz bereits 3 Jahre später durch die Novellierung des BauGB-Maßnahmen-G ersetzt.

16 Bundesstiftung Aufarbeitung: A. Friszke, Widerstand und Opposition im Kommunismus 1945-91, Berlin 2016, S. 3: Untergrundkämpfe waren u.a. keine Seltenheit, https://www.dissidenten.eu/fileadmin/user_upload/Polen/Oppositionsgeschichte/dissidenten_eu/Polnische-Oppositionsgeschichte.pdf (abgerufen: 27.03.2021).

17 Bundesstiftung Aufarbeitung: ibidem, S. 1, 2: Untergrundkämpfe waren u.a. keine Seltenheit, https://www.dissidenten.eu/fileadmin/user_upload/Polen/Oppositionsgeschichte/dissidenten eu-Polnische-Oppositionsgeschichte.pdf (abgerufen: 27.03.2021).

18 J. Schneider, Die Ursachen für den Zusammenbruch der Sowjetunion und der DDR (19451990), Stuttgart 2017, S. 28, 201.

19 G. Winter, K. Kalichava, Rechtstransfer und Eigendynamik in Transformationsländern, „ZaöRV“ 79, 2019, S. 294: Hier ist kein spezieller Bezug auf Polen gesetzt, sondern es geht um generelle Probleme innerhalb der Länder, die sowjetisch-geprägt wurden.

20 O. Schwarzer, Sozialistische Zentralplanwirtschaft in der SBZ/DDR, Stuttgart 1999, S. 184 ff.

21 G. Winter, K. Kalichava, op. cit., S. 295: Hier ist kein spezieller Bezug auf Polen gesetzt, der Fokus liegt auf den generellen Festlegungen bzgl. des Umgangs mit dem sozialistischen Eigentum in sowjetisch-geprägten Ländern. 
tischen Festlegungen waren augenscheinlich keine wertstiftenden, sozialliberalen Bedarfsplanungen möglich. Staatlich regulierte Bauprozesse, die durch staatliche Behörden bis ins Detail geplant wurden, hatten Verfahrensverzögerungen zur Folge, ${ }^{22}$ die der Entwicklung nicht dienlich waren. Nach dem Zusammenbruch der Sowjetunion löste sich Polen aus dem Modell der Planwirtschaft und implementierte peu à peu eine Marktwirtschaft nach westlichem Vorbild. ${ }^{23}$ Die Hinwendung zu einer sich den Märkten öffnenden Ökonomie brachte einen signifikanten, wirtschaftlichen Boom, der sich in der Entwicklung des Bruttoinlandprodukts widergespielte: von 1990 bis 2000 gab es ein BIP-Wachstum von ca. $175 \%{ }^{24}$ Die ab den 1970ern stetig gewachsene, planungsrechtliche Dynamik, die in Deutschland v.a. durch das Raumordnungsgesetz (ROG) manifestiert wurde, entwickelte sich in Polen erst nach der Wende. So besagt u.a. Art. 87 Abs. 2 des polnischen Raumplanungs/-ordnungsrecht (ustawa o planowaniu i zagospodarowaniu przestrzennym ${ }^{25}$ ), welches in den 2000ern bereits mehrfach durch Verordnungen ergänzt und korrigiert wurde, ${ }^{26}$ dass raumwirtschaftliche Festlegungen, die vor dem 1. Januar 1995 erlassen wurden, spätestens am 31. Dezember 2003 ungültig werden sollen. Anhand dieses Gesetzesbeispiels zeigt sich deutlich, wie jung die planungsrechtliche Entwicklung Polens ist, da diese erst nach 1990 auf Basis von einer westlich-geprägten Marktwirtschaft vorangetrieben wurde. Von nationaler Ebene ausgehend, bilden den wohl wesentlichstenhistorisch begründeten - Unterschied die ungleichen Staatsstrukturen, die offenkundig auf die planungsrechtlichen Entwicklungen jeweils einen erheblichen Einfluss hatten. Deutschland wies nach dem zweiten Weltkrieg einen Staat auf, der durch ein föderalistisches System geprägt wurde; dessen Zweck ist es im Sinne eines Machtsplittings, die einzelnen Bundesländer mit mehr Eigenständigkeit zu belegen als den Bundesstaat im Ganzen. ${ }^{27}$ Die Auswirkungen lassen sich v.a. im Bereich der Gesetzgebung, wo

${ }^{22}$ K. Steinitz, Die Wirtschaftsplanung in der DDR - Probleme, Erfahrungen, Schlussfolgerungen, [in:] Erfahrungen und Probleme der Planung und der wirtschaftlichen Entwicklung in der $D D R$, Schlussfolgerungen für die Zukunft, hrsg. Rosa-Luxemburg-Stiftung, Werder an der Havel 2007, S. 11: Zeitliche Verzögerungen von wirtschaftlichen Verfahren waren in vielen kommunistischen, von der Sowjetunion kontrollierten, Ländern zu erkennen. In dieser Abhandlung war dies anhand der wirtschaftlichen Nachteile innerhalb der DDR erkennbar.

23 R. Ulrich, Der Übergang zur Marktwirtschaft in der CSFR, Polen und Ungarn: Ausgangsbedingungen und erste Schritte, Berlin 1991, S. 1.

${ }^{24}$ Statista, Polen: Bruttoinlandsprodukt von 1980 bis 2019, de.statista.com - Statistik - Internationale Länderdaten - Europa - Branche durchsuchen - Bruttoinlandsprodukt Polen - Suchergebnis Bruttoinlandsprodukt (BIP) von Polen 2025.

25 P. Rataj, Die Grundzüge des polnischen Planungsrechts, [in:] Die Grundzüge des polnischen Planungsrechts, hrsg. Botschaft der Republik Polen in der Bundesrepublik Deutschland, Berlin 2012, S. 7: Das Gesetz ist vom 27.03.2003.

26 Ibidem.

27 J. Isensee, Idee und Gestalt des Förderalismus im Grundgesetz, [in:] Handbuch des Staatsrechts, Bd. VI, hrsg. P. Kirchhof, Heidelberg 2008, Rdn. 14 ff.; Wissenschaftliche Dienste des Deutschen Bundestags, Dokumentation: Aufbau eines föderalistischen Staatswesens, Berlin 2019, S. 4. 
die Länder eindrücklich mitbestimmen, ${ }^{28}$ erkennen. Polen wiederum ist seit seiner Verfassung von 1997 de iure ein Zentralstaat. ${ }^{29}$ Es gibt zwar in Polen ebenso Verwaltungsapparate, die sog. Woiwodschaften, ${ }^{30}$ doch sind diese, anders als in Deutschland, nicht an der Gesetzgebung beteiligt und übernehmen im weitesten Sinne lediglich Aufgaben verwaltungstechnischer Natur. ${ }^{31}$ Auf supranationaler Ebene ist festzustellen, dass sowohl Deutschland, das bereits 1951 Gründungsmitglied der Gemeinschaft für Kohle und Stahl war, als auch Polen, das in die damalige Europäische Gemeinschaft (EG) 2004 eingetreten ist, beide Mitglied der Europäischen Union ${ }^{32}$ sind. $^{33}$

Der hier nun kurz skizzierte, geschichtliche Grundriss soll verdeutlichen, durch welch unterschiedliche Startpunkte und Entwicklungsstrukturen Deutschland sowie Polen politisch wie auch rechtlich gekennzeichnet sind. Im Folgenden wird nun jeweils separat dargestellt, welche planungsrechtlichen Grundstrukturen in diesen beiden Ländern bestehen und welchen Einfluss durch internationale ${ }^{34}$ und supranationale Vorgaben bestehen. Abschließend wird ein direkter Rechtsvergleich beider Länder zu einem besseren, gegenseitigen Verständnis herangezogen.

\section{B. Raumnutzung auf unterschiedlichen Ebenen}

Um sich dem vielschichtigen Thema der Raumnutzung zu nähern, empfiehlt es sich zunächst, die einzelnen, notwendigen Ebenen zu betrachten. Zunächst sind dort die einzelstaatlichen Interessen Deutschlands und Polens zu nennen. Darüber hinaus kommen völkerrechtliche Verträge zwischen einzelnen Ländern in Betracht. Nimmt man dabei beide Länder in den Blick, ${ }^{35}$ darf die europäische Dimension der Raumordnung nicht unerwähnt bleiben. Deutschland war ein Gründungsmitglied der EGKS. Aus dieser Gemeinschaft ist die Europäische Union

28 Siehe hierzu: Art. 70 ff. GG: Die Länder haben grundsätzlich das Recht der Gesetzgebung inne.

29 K. Bachmann, Zur Entwicklung der polnischen Demokratie, „APuZ - Zeitschrift der Bundeszentrale für politische Bildung“"10-11, 2018, S. 10.

${ }^{30}$ P. Rataj, op. cit., S. 10.

31 K. Bachmann, op. cit., S. 10: Ein eigener zu verwaltender Haushalt und ein installiertes Steueraufkommen sind zwar vorhanden, doch eine direkte Einflussnahme auf die Erlassung von Gesetzen haben die Woiwodschaften - Stand heute - nicht.

32 Stand 2021.

33 O. Schmuck, G. Unser, Zeitbilder: Die Europäische Union, Bonn 2018, S. 8.

34 u.a. UN-Vorgaben: s. S. Baumann et al., 1987-2017: 30 Jahre Montrealer Protokoll, Dessau-Roßlau2017, S. 5 ff.: Zu erwähnen ist, dass seit dem 1987 verabschiedeten Montrealer Protokoll, was v.a. auf internationaler Ebene das Verbot von u.a. FCKW-Stoffen nach sich zog, der Umweltschutzgedanke immer mehr auf international-rechtlicher Ebene implementiert wurde. Im Weiteren wird noch näher darauf eingegangen.

35 Europäische Gemeinschaft für Kohle und Stahl. 
entstanden. In diese ist Polen am 1. Mai 2004 eingetreten. Grundsätzlich hat jedes Beitrittsland dabei den „Acquis communautaire“36 zu akzeptieren, Art. 49 des Vertrages über die Europäische Union, ${ }^{37}$ so dass auch die Regelungen, welche vor 2003 erlassen worden sind, für Polen relevant sind. ${ }^{38}$

\section{Raumplanung in Deutschland}

Die Verfassung der Bundesrepublik Deutschland ist das Grundgesetz, welches seinerseits fünf Staatsstrukturprinzipien ${ }^{39}$ kodifiziert. Darin sind die horizontale Gewaltenteilung (rechtsetzende Gewalt, rechtsprechende Gewalt und ausführende Gewalt) und die vertikale Gewaltenteilung (Bundesrepublik Deutschland und die 16 Bundesländer) vorgesehen. Auch im Rahmen der Raumplanung gilt zunächst, dass die Verwaltungs- und Rechtsetzungskompetenz von den Ländern ausgeht, Art. 30, 70 GG.

Die Landesplanung in Deutschland ist auf verschiedenen Ebenen zu finden. Zunächst bestehen Planungsmöglichkeiten für das gesamte Bundesgebiet. Bei raumbedeutsamen Planungen bestehen Zuständigkeiten für die Fachplanung nach Sondergesetzen und auf Ebene der Gemeinden ist die eigentliche Bauleitplanung beheimatet. Dabei stehen diese Bauleitplanungen auf gemeindlicher Ebene in einem Spannungsverhältnis zu anderen Planungen. ${ }^{40}$

Bezüglich der Raumordnung in Deutschland ist zunächst auf das Raumordnungsgesetz einzugehen. Bei dieser bundesrechtlichen Regelung gilt seit der Föderalismusreform 2006, dass hierfür nicht mehr die Rahmengesetzgebungskompetenz gilt (ehemals Art. 75 GG). Insoweit ist die Raumordnung seither der konkurrierenden Gesetzgebung (Art. 74 Nr. 31 GG) untergliedert. Diese Veränderung führt dazu, dass nunmehr nicht mehr begründet werden muss, warum ein Bundesgesetz erforderlich ist. Mithin begründet sich zunächst eine Zuständigkeit des Bundesgesetzgebers. Die Länder haben in diesem Kontext jedoch ein Abweichungsrecht (Art. 70 III Nr. 4 GG). Möglich ist eine landeseigene Regelung, sofern der Bund von seiner Gesetzgebungszuständigkeit keinen Gebrauch gemacht hat. Exemplarisch sei insoweit auf einzelne Landesplanungsgesetze der Länder verwiesen. Bei einer Divergenz zwischen Bundes- und Landesrecht gilt Art. 31 GG.

Die erste bundesrechtliche Regelung, das ROG, erfolgte auf Grundlage der damaligen Rahmengesetzgebung am 8. April 1965. ${ }^{41}$ Es gilt nach Art. 125a I 1 GG fort. Nach der grundsätzlich denkbaren Regelung durch die Länder,

\footnotetext{
36 Als „Acquis communautaire“ wird das gesamte Regelungsgefüge der EU bezeichnet.

37 Vertrag über die Europäische Union (konsolidierte Fassung), URi.

38 Gesetzgebung - EU-Info.de (eu-info.de).

39 Föderalismus, Rechtsstaatlichkeit, Demokratie, Sozialstaatlichkeit, Republik.

40 B. Stüer, Handbuch des Bau- und Fachplanungsrechts, München 2015, Rn. 99.

41 BGBl. I, 306.
} 
Art. 125a I 2 GG i.V.m. Art. 72 III GG, wurde am 22. Dezember 2008 das Gesetz zur Neufassung des Raumordnungsgesetzes und zur Änderung anderer Vorschriften erlassen. Innerhalb des ROGs wurde u.a. das Herzstück des deutschen Raumordnungsrechts verankert, das sog. Gegenstromprinzip. ${ }^{42}$ Die übergreifende Überlegung in diesem Rahmen basiert auf der Vorstellung, dass der Gesamtraum der Bundesrepublik Deutschland und seine Teilräume u.a. durch Raumordnungspläne und diesbezügliche Abstimmungen zu strukturieren und zu sichern sind. ${ }^{43}$ In der Planung des nationalen Gesamtbereiches sollen die räumlichen Gegebenheiten und Anforderungen seiner untergliederten Teilgebiete notwendigerweise in Betracht gezogen werden. ${ }^{44}$

Mit Rücksichtnahme auf dieses Prinzip ist festzustellen, dass mithin im Bereich der Außenwirtschaftszonen ${ }^{45}$ in der Nord- und der Ostsee dem Bund nach jedenfalls unbeschränkte Rechtsetzungskompetenz zukommt. In der Nord- und Ostsee ist die deutsche Außenwirtschaftszone im Wesentlichen mit dem sogenannten deutschen Festlandsockel identisch. Kollidieren benachbarte Außenwirtschaftszonen miteinander, so werden die Grenzen vertraglich festgelegt. ${ }^{46} \mathrm{Im}$ Falle von Deutschland und Polen sind im Rahmen der Beschlüsse der Potsdamer Konferenz vom 2. August 1945 Grenzverläufe bestimmt worden, welche mit dem Warschauer Vertrag vom 7. Dezember 1970 nochmals bestätigt worden sind. ${ }^{47}$ Eine grenzüberschreitende Abstimmung von raumbedeutsamen Planungen und Maßnahmen ist im deutschen Planungsrecht vorgeschrieben. ${ }^{48}$

Die Außenwirtschaftszonen müssen dabei die Sicherheit und Leichtigkeit des Schiffsverkehrs gewährleisten, können wirtschaftlich nutzbar gemacht werden, sind zur Erlangung wissenschaftlicher Erkenntnisse unabdingbar und müssen mit Blick auf die Meeresumwelt besonders beachtet werden. Gerade vor dem Hintergrund zu erwartender klimatischer Veränderungen sind Anpassungsabsichten bezüglich der Raumordnung in den Koalitionsvertrag aufgenommen worden. ${ }^{49}$ Vor Inkrafttreten des GeROG dienten das Seeaufgabengesetz von 1965, die Seenanlagenverordnung von 1997 und die Bundesberggesetze als Rechtsgrundlagen.

$42 \S 1$ III ROG.

$43 \S 1$ I S. 1 ROG.

44 Abgeleitet aus der Legaldefinition des Gegenstromprinzips in § 3 III ROG.

45 Erstmals so verwendet im UN-Seerechtsübereinkommen von 1982.

46 Nordsee: Niederlande, Großbritannien und Dänemark; Ostsee: Dänemark, Schweden, Polen.

47 Art. 1 I des völkerrechtlichen Vertrages zwischen der Bundesrepublik Deutschland und der Volksrepublik Polen über die Grundlagen der Normalisierung ihrer gegenseitigen Beziehungen vom 7. Dezember 1970.

48 Projekte wurden in diesem Rahmen durch die Gemeinschaftsinitiative des Europäischen Fonds für regionale Entwicklung (EFRE) INTERREG III gesponsert.

49 Koalitionsvertrag zwischen CDU, CSU und SPD vom 7.2.2018, dort Ziffer XI. 2, https:// www.zeit.de/politik/deutschland/2018-02/koalitionsvertrag.pdf?utm_referrer $=$ https $\% 3 \mathrm{~A} \% 2 \mathrm{~F} \%$ 2Fwww.google.com\%2F (abgerufen: 27.03.2021). 


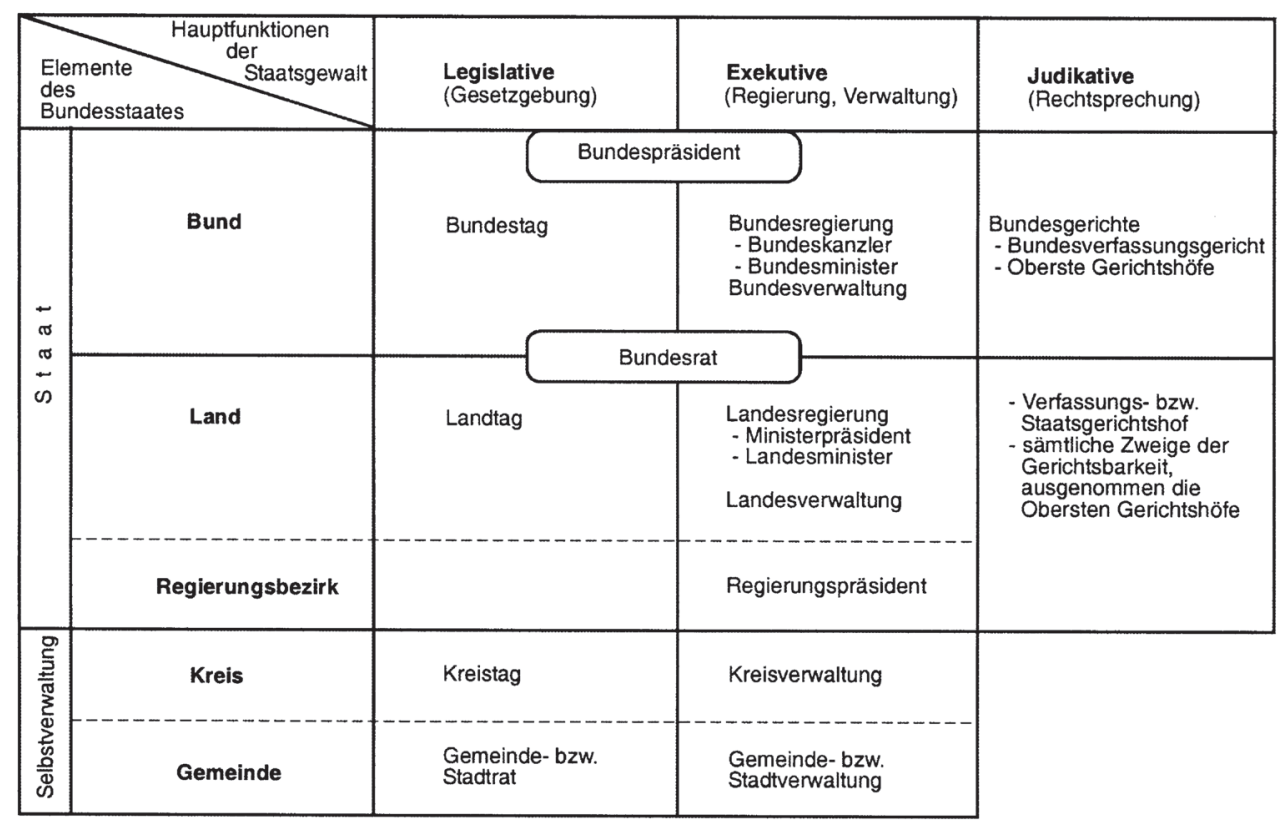

Gerd Turowski, Dortmund 1999

Quelle: Z. Niewiadomski, G. Turowski, Deutsch-Polnisches Handbuch der Planungsbegriffe. Polsko-niemiecki leksykon pojęć planistycznych, Hannover-Warschau 2001, S. 131.

\section{Raumplanung in Polen}

Aus polnischer Sicht ist Deutschland seit vielen Jahren der wichtigste Handelspartner; im Gegenzug ist Polen - aufgrund seiner Exporte - auch regelmäßig unter den zehn umsatzstärksten Handelspartnern Deutschlands. ${ }^{50}$ Damit einher geht auch eine zunehmende Annäherung der öffentlich-rechtlichen Strukturen. Wie in Deutschland wird in Polen die Baufreiheit als Ausdruck der durch die Verfassung (GB1.: (Dz.U.) 1997 Nr. 78, Pos. 483 m.Ä.) geschützten Werte, wie der Freiheit des Menschen, Art. 31 I der Verfassung der Republik Polen, und des Eigentums, Art. 21 I der Verfassung der Republik Polen, verstanden; der Grundsatz der Baufreiheit ergibt sich aus Art. 4 der Bauordnung der Republik Polen, wonach jeder ein Recht zur Bebauung eines Grundstücks hat, wenn er sein Verfü-

502020 belegte Polen in einem Statista-Ranking Platz 6, mit insgesamt 64,7 Milliarden Euro Exportvolumen. Statista: Rangfolge der wichtigsten Handelspartner Deutschlands nach Wert der Exporte im Jahr 2020, https://de.statista.com/statistik/daten/studie/2876/umfrage/rangfolge-derwichtigsten-handelspartner-deutschlands-nach-wert-der-exporte/\#: :text=Bezogen $\% 20$ auf $\% 20$ die\%20Exporte\% 20waren,95\%2C98\%20Milliarden\%20Euro (abgerufen: 27.03.2021); N. Becker, Deutaschland bleibt Polens wichtigster Handelspartner, GTAI Wirtschaftsumfeld, Website von GTAI - Menü - Trade - Wirtschaftsumfeld - Berichte Wirtschaftsumfeld; Statista: Wichtigste Handelspartner (Exporte) für Deutschland 2019 | Statista. 
gungsrecht über das Grundstück zu Bauzwecken nachweist und das Bauvorhaben mit den Vorschriften im Einklang steht. ${ }^{51}$ Dabei ist die Planung in Polen dreistufig aufgeteilt. In dem unitarischen Staat wird unterschieden zwischen der Landesplanung (Raumplanung im Bereich des ganzen Landes), der Regionalplanung (Woiwodschaftsplanung) und Ortsplanung. ${ }^{52}$ Diese Untergliederung geht auf ein entsprechendes Gesetz aus dem Jahr 1998 zurück (GB1.: (Dz.U.) Nr. 96 Pos. 603). Im Sinne dieses Gesetzes wurden ab dem 1. Januar 1999 die 16 Woiwodschaften geschaffen, wobei die staatliche Verwaltung in diesen durch das Gesetz über die staatliche Verwaltung in der Woiwodschaft vom 5. Juni 1998 (GB1.: (Dz.U.) Nr. 91 Pos. 577) geregelt wird. Die Ziele der staatlichen Raumentwicklungspolitik werden in Form der Nationalen Raumbewirtschaftungskonzepte (Koncepcja przestrzennego zagospodarowania kraju) beschlossen. ${ }^{53}$ Raumwirtschaft wird dabei in Polen als Oberbegriff der räumlichen Organisation von gesellschaftlichen Systemen sowie der Gestaltung der räumlichen Organisation, Struktur und Funktionsfähigkeit von territorialen Gesellschaftssystemen als Tätigkeit verstanden. ${ }^{54}$

Außerdem gab es ein Gesetz über die Raumbewirtschaftung (ustawa o zagospodarowaniu przestrzennym) aus dem Jahr $1994,{ }^{55}$ das das zentralisierte und hierarchische Raumplanungssystem der Volksrepublik beseitigte und die Gemeinden in die Lage versetzte, in bedeutendem Maße die Raumbewirtschaftung des Landes zu steuern. ${ }^{56}$ Auf dieser Grundlage wurden Raumordnungspläne für Woiwodschaften erarbeitet, insbesondere für Großpolen. Weiter gab es 2000 ein Gesetz zur Förderung der Regionalentwicklung. Im Vorfeld zum Beitritt Polens zur EU wurde aufgrund zahlreicher erkannter praktischer Schwächen dieses Gesetz durch das Gesetz über Raumplanung- und -bewirtschaftung (ustawa o planowaniu i zagospodarowaniu przestrzennym) im Jahr 2003 abgelöst. ${ }^{57}$ Zahlreiche Novellierungen erfolgten, änderten jedoch nichts an den kodifizierten Grundzügen. ${ }^{58}$ Seither ist das Regelungssystem mit dem deutschen Raumordnungsrecht konzeptuell durchaus vergleichbar. ${ }^{59}$

51 P. Ostojski, W. Piątek, Legalisierung von illegalen baulichen Anlagen in Polen und Deutschland, „Landes- und Kommunalverwaltung“ 4, 2014, S. 145 m.w.N.

52 S. Misztal, Landesplanung in Polen, „Plan: Zeitschrift für Planen, Energie, Kommunalwesen und Umwelttechnik“ 6, 1963, S. 206 (207); A. Tölle, Stadt- und Raumentwicklung Polen, [in:] Handwörterbuch der Stadt- und Raumentwicklung, hrsg. ARL, Hannover 2018, S. 2317 (2318).

53 A. Tölle, op. cit., S. 2317 (2320).

54 S. Ebert, A. Tölle, M. Wdowicka, Planung in Deutschland und Polen aus kommunaler Perspektive - Planowanie $w$ Polsce $i$ w Niemczech z perspektywy gminy, Hannover-Poznań 2012, S. 3.

55 A. Tölle, op. cit., S. 2317 (2318).

56 S. Ebert, A. Tölle, M. Wdowicka, op. cit., S. 8.

57 A. Tölle, op. cit., S. 2317 (2318); S. Ebert, A. Tölle, M. Wdowicka, op. cit., S. 9.

58 S. Ebert, A. Tölle, M. Wdowicka, op. cit., S. 9.

59 S. Slapa et al., Abstimmungsprozesse in Grenzregionen- Der Umgang mit Ansiedlungen in den Grenzregionen Deutschland-Polen und Deutschland-Tschechien, Berlin 2006, Ziffer 1.1. 
Nachfolgendes Schema mag die Entscheidungsebenen und -institutionen im polnischen Verwaltungssystem deutlich machen:

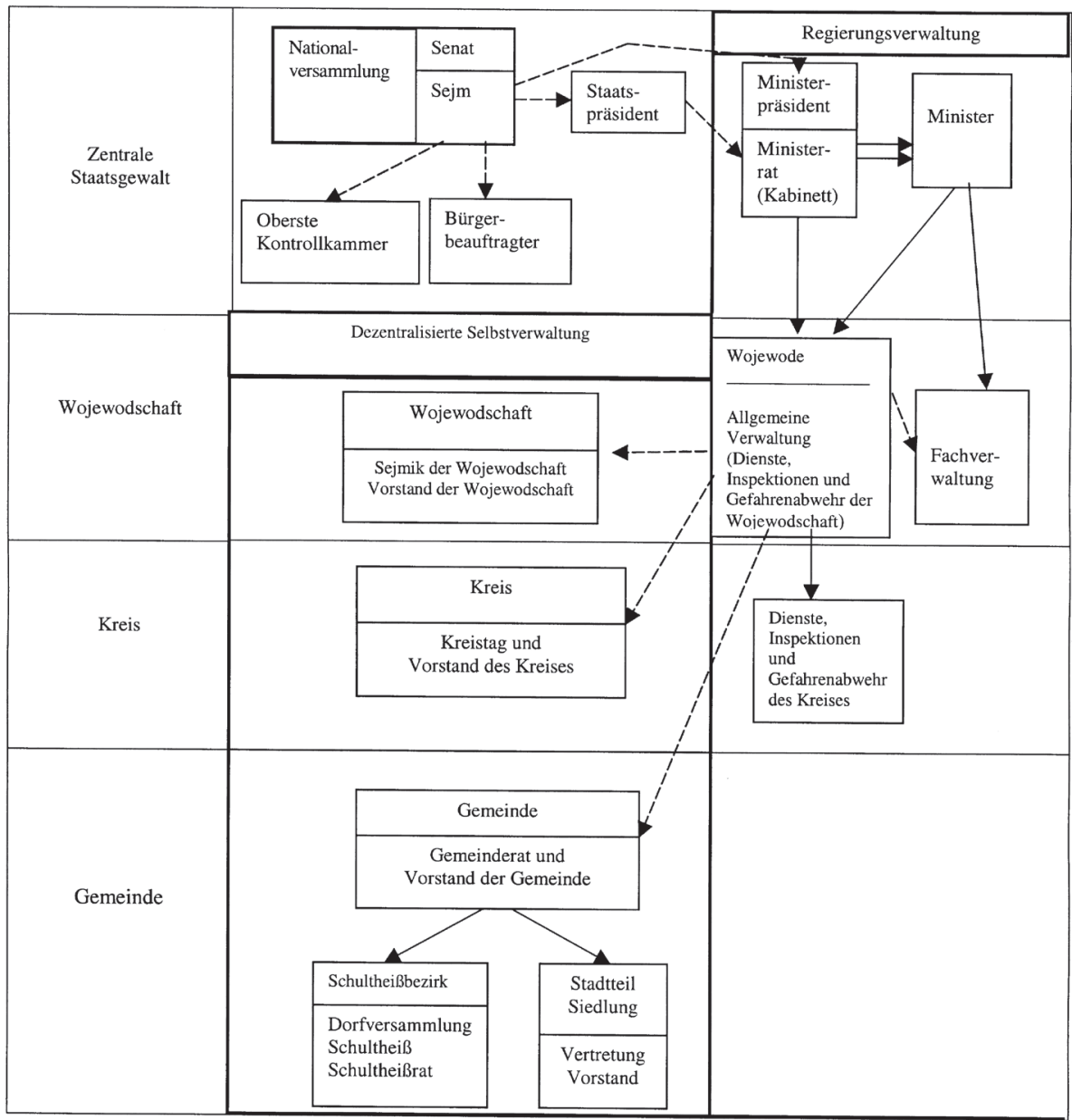

Funktionale Verbindungen

Autor: Zygmunt Niewiadomski

Organisatorisches Unterstellungsverhältnis

Quelle: Z. Niewiadomski, G. Turowski, op. cit., S. 30.

\section{Deutsch-polnische Zusammenarbeit}

Die entwickelten Leitbilder zielen darauf ab, den ländlich geprägten OderNeiße-Raum mit seiner geringen Bevölkerungsdichte und der einzigartigen Natur- und Landschaftsvielfalt zu einem Gebiet mit funktionierender Siedlungs- 
struktur und engen Verkehrsverbindungen zu entwickeln. ${ }^{60}$ Der Grenzraum wird als räumliche Einheit gesehen. ${ }^{61}$

Der ,eigentliche Grenzraum“ verfügt mit Settingen über ein überregionales Zentrum. Stadtregionen, die das „Deutsch-Polnische Haus“ bilden, müssen, um sich als europäische Wirtschaftsregion zu positionieren, gezielte, politische und grenzübergreifende Anstrengungen unternehmen. Dass dieser Ansatz in der Praxis verfolgt wird, zeigt sich insbesondere anhand verschiedener Projekte (wie das MORO-Projekt ${ }^{62}$ ) oder Einrichtungen (wie das Deutsch-Polnische-Raumordnungsportal ${ }^{63}$ ).

Nach einer Verwaltungsreform sank ab dem 1. Januar 1999 die Zahl der Woiwodschaften von 49 auf 16, was günstigere Bedingungen für die Zusammenarbeit im Grenzraum geschaffen hat. ${ }^{64}$

\section{Länderübergreifende Raumplanung}

Hier ist v.a. die grenzübergreifende Euroregion Pomerania ${ }^{65}$ zu nennen. Fördermittel aus dem europäischen Strukturfonds sind für dieses Gebiet von großer Bedeutung. ${ }^{66}$

Neben den dargestellten Projekten und Verfahrensabläufen ergeben sich auch aufgrund der Mitgliedschaft zur Europäischen Union einige Vorgaben. Diese ist, gemäß Art. 4 II des Vertrages über die Arbeitsweise der Europäischen Union mit den Mitgliedstaaten, unter anderem in den Bereichen der Landwirtschaft und Fischerei, der Umwelt, dem Verkehr, transeuropäischer Netze und der Energie zuständig. Weiterhin wurde ihr im Wege der begrenzten Einzelermächtigung, Art. 5 des Vertrages über die Europäische Union, die ausschließliche Zuständig-

${ }^{60}$ W. Selke, Deutsch-Polnisches Haus: Auf dem Wege zu einer grenzüberschreitenden Wirtschaftsregion in Mitteleuropa?, [in:] Strukturwandel in Ostdeutschland und Westpolen, hrsg. M. Stroll, Hannover 2004, S. 2.

61 Ibidem, S. 5.

${ }^{62}$ Bundesministerium für Verkehr, Bau und Stadtentwicklung, Raumordnerische Zusammenarbeit im deutsch-polnischen Grenzraum, 2012, Bundesinstitut für Bau-, Stadt und Raumforschung - Suchleiste „Raumordnerische Zusammenarbeit im deutsch-polnischen Grenzraum“; MORO-Projekt „Raumbeobachtung Deutschland und angrenzende Regionen“, Website von SIG-GR.eu - Kooperationen und Projekte - MORO-Projekt „Raumbeobachtung in Deutschland und angrenzenden Regionen".

${ }^{63}$ Deutsch-polnisches Raumordnungsportal/Polsko-Niemiecki Portal Gospodarki Przestrzennej.

${ }^{64}$ S. Slapa et al., op. cit., Ziffer 1.1.

${ }^{65}$ Die die historische Einheit von Vorpommern und Hinterpommern nachzeichnet. Vgl. dazu etwa W. Wagner, EU-Millionen für Straßen in Grenzregion zu Polen, „Schweriner Volkszeitung“ 2016. Website der Schweriner Volkszeitung - über die Suchleiste „EU-Millionen für Straßen in Grenzregion zu Polen".

66 S. Ebert, A. Tölle, M. Wdowicka, op. cit., S. 8. 
keit bezüglich der Erhaltung der biologischen Meeresschätze im Rahmen der gemeinsamen Fischereipolitik und im Rahmen der gemeinsamen Handelspolitik übertragen. Im Zuge dessen haben sowohl Deutschland als auch Polen Leitbilder definiert, welche den Gedanken des Europäischen Raumentwicklungskonzepts aufgreifen, ein dezentrales System hoch entwickelter Wirtschaftsregionen zu fördern und untereinander zu vernetzen. ${ }^{67}$ Aufgrund der zentralen Lage der beiden Länder bestehen auch übergreifende europäische Verkehrsplanungen. So bilden die Städte Berlin, Warschau (Warszawa), Minsk, Moskau und Nischni Nowgorod den paneuropäischen Korridor II und Berlin, Dresden, Breslau (Wrocław) und Kiew den paneuropäischen Korridor III. ${ }^{68}$

Eine deutliche Aufwärtsentwicklung erlebte der Planungsgedanke durch den Aufschwung des Ökologiegedankens in den 80er und 90er Jahren, der v.a. durch europarechtliche Impulse in die Wege geleitet wurde. ${ }^{69}$ Dabei ist zu beachten, dass Regelungen zugunsten der Umwelt sowohl das Genehmigungsverfahren spezieller Vorhaben betreffen als auch ihrerseits die planbare Fläche einschränken können. ${ }^{70}$ Der Umweltverträglichkeitsgedanke wird zum zentralen Aspekt des Planungsrechts ${ }^{71}$ sowohl im Raum- als auch im Fachplanungsrecht. Der Erlass des Gesetzes über die Einführung einer Umweltverträglichkeitsprüfung (UVPG) und die Umsetzung der (bereits erwähnten) Richtlinie über die strategische Umweltprüfung (SUPG) manifestierte diesen Aspekt auf rechtlicher Ebene.

\section{Raumbedeutsame Projekte}

Im Folgenden soll durch Beispiele aus den Bereichen Energiewirtschaft und Infrastruktur die Raumordnungspraxis in Deutschland und Polen konkretisiert werden.

\section{Offshore-Windparks in Deutschland und Polen}

Das erste Beispiel mit planungs- und baurechtlicher Relevanz sind OffshoreWindparks im Ostseeraum. Offshore-Windanlagen, die aus in Küstennähe im Meer installierten Windkraftanlagen bestehen, sind einerseits gekennzeichnet durch ihre hohe Effizienz, da an ungefähr 340 Tagen innerhalb eines Jahres Strom produziert

67 W. Selke, op. cit., S. 7.

${ }^{68}$ Ibidem, S. 12.

69 Am Beginn steht die EG-Vogelschutzschutzrichtlinie.

70 Exemplarisch sei insoweit auf das „Natura-2000“-Netz verwiesen. Im Zuge dieses Projektes sollten die Mitgliedstaaten Naturschutzgebiete ausweisen, deren spätere anderweitige wirtschaftliche Nutzung damit unmöglich gemacht wird, Homepage der Europäischen Kommission - Grundlegendes - Schutzgebiete in der EU - Natura 2000 (abgerufen: 27.03.2021).

71 D. Dörr, op. cit., § 38, Rdn. 12 ff. 
werden kann, und andererseits durch eine ziemlich akkurate Vorhersagbarkeit, was die von ihnen zu erzeugenden Stromerträge anbelangt. ${ }^{72}$ Aufgrund der markanten Lage der Ostsee werden zwar teilweise gemäßigtere Windgeschwindigkeiten als in der Nordsee gemessen, doch sind diese mit $9,7 \mathrm{~m} / \mathrm{s}$ in einer Messhöhe von $100 \mathrm{~m}$ immer noch beträchtlich. ${ }^{73}$ Durch diese Werte bedingt, ist es nicht verwunderlich, dass in der Ostsee, vor allem an deutscher Küste bereits einige Offshore-Windparks in Betrieb sind. ${ }^{74}$ Auch auf polnischer Seite wird, aufgrund der oben genannten Vorteile der Offshore-Windanlagen und der im Jahre 2017 verabschiedeten Strategie für die Entwicklung des Brennstoff- und Energiesektors (PEP2040) ${ }^{75}$, die Einführung von Windanlagen in der Ostsee noch stärker als zuvor in den Blick genommen. Im Rahmen dieser Strategie wurden auf rechtlicher Ebene Vorkehrungen getroffen, die den Bau solcher Anlagen in Küstennähe für Investoren wirtschaftlich erleichtern. ${ }^{76}$ Ein neues Gesetz etabliert ein Differenzvertragsmodeli, ${ }^{77}$ welches den Betreibern von Windkraftanlagen die Möglichkeit bietet, negative Einnahmedifferenzen, die im freien Stromhandel aufgrund von Marktschwankungen entstehen können - nach einem festen Vergütungsatzeinzufordern.Diese Vergütung wird durch staatliche Finanzierung bereitgestellt. ${ }^{78}$ Durch die neue Absicherung für Investoren dieser Energiequellen soll gewährleistet werden, dass in Polen bis 2025 die ersten geplanten Anlagen in Betrieb genommen werden und bis zum Jahre 2040 bereits eine elektrische Leistung in Höhe von 8-11 Gigawatt - rein durch Windkraftanlagen in der Ostsee - erzeugt wird. ${ }^{79}$ Die formellen Verfahren sind durch diese finanziellen Absicherungen auf Investorenseite nicht gänzlich verschwunden. Planungsrechtliche sowie baurechtliche Regelungen sind weiterhin zu

72 K. Rohrig et al., Energiewirtschaftliche Bedeutung der Offshore-Windenergie für die Energiewende, Berlin 2013, S. 6.

${ }^{73}$ Homepage des Bundesministeriums für Wirtschaft und Energie - Forschung - Windenergie auf See Windklimatologie (abgerufen: 27.03.2021).

74 So z.B. der bereits im Jahre 2011 in Betrieb genommene Windpark EnBW Baltic 1, der 16 km vor der Küste Mecklenburg-Vorpommerns liegt: Flyer Windpark EnBW Baltic 1 - Der erste kommerzielle Offshore-Windpark Deutschlands.

75 Energieministerium Warschau, Zusammenfassung und Schlussfolgerungen der Umweltprüfung der polnischen Energiepolitik bis zum Jahr 2040, S. 5: Die Klima- und Energiepolitik seitens der Europäischen Union, insbesondere das im Jahre 2009 verabschiedete $3 \times 20 \%$ - Paket hatten u.a. auf diese Strategieentwicklung Einfluss.

76 Am 21. Januar 2021 wurde das Gesetz zur Förderung der Stromerzeugung in Windparks auf dem Meer (Ustawa z dnia 17 grudnia 2020 o promowaniu wytwarzania energii elektrycznej $w$ morskich farmach wiatrowych) vom polnischen Staatspräsidenten, Andrzej Duda, unterzeichnet und trat im Februar 2021 in Kraft.

77 Basierend auf dem britischen Modell des Contracts for Differences.

78 Art. 4 des Gesetzes Ustawa o promowaniu wytwarzania energii elektrycznej w morskich farmach wiatrowych, der darauf hinweist, dass eine negative Saldodeckung unter bestimmten Bedingungen möglich ist.

79 A. Ceglarz, Die polnische Energiepolitik, Warszawa 2020, S. 19; T. Weber, Warschau schickt Windkraft in die See, Website der „Erneuerbare Energien“ (abgerufen: 27.03.2021). 
beachten. Im Fall des Battyk Środkowy $I I I^{80}$ ist hier ein Vorgehen in vier Schritten zu erkennen. Im März 2012 wurde die Genehmigung für eine künstliche Insel, als Fundament der Anlage, eingeholt, danach besteht insgesamt zehn Jahre ${ }^{81}$, d.h. bis 2022, die Möglichkeit eine Baugenehmigung für die Anlage zu erhalten; der Baubeginn selbst darf maximal drei Jahre dauern. ${ }^{82}$ Spätestens fünf Jahre nach Baubeginn muss die Nutzung der künstlichen Insel initiiert werden. ${ }^{83}$ Darauffolgend werden umweltrechtliche Genehmigungen, die für eine Fülle an verschiedensten Windkraftanlagen notwendig sind, auch für Offshore-Windkraftanlagen vorgeschrieben. ${ }^{84}$ Umweltrechtliche Faktoren, die generell in diesem Bereich zu berücksichtigen sind, sind u.a. Aspekte des Lärmschutzes, des Schutzes der Flora und Fauna und des Schutzes der Kulturlandschaft. ${ }^{85}$ Der Lärmschutz ist - aus erstmal nicht von Vornherein ersichtlichen Gründen - nicht zu unterschätzen. Ein Großteil der Meerestiere haben ein Gehör, was auf Schallnutzung basiert. ${ }^{86}$ Windkraftanlagen können diese Meerestiere orientierungslos machen. ${ }^{87}$ Der geplante Standort ist demnach ein entscheidender Aspekt. Darüber hinaus ist auch der Schutz der Flora und Fauna ein nicht zu unterschätzender Umstand, selbst im Meeresbereich. Untersuchungen im Belwind Wind Park ${ }^{88}$ haben einerseits gezeigt, dass zwar heimische Vögel, wie z.B. der nördliche Tölpel oder die gewöhnliche Trottellumme, um über 70-80\% zurückgegangen sind, andere Vogelarten, wie bspw. die Heringsmöve, einen Zuwachs von über $430 \%$ erfahren hat. ${ }^{89}$ Solche Analysen zeigen, dass die umweltrechtlichen Voraussetzungen im Rahmen solcher Großprojekte nicht unterschätzt werden dürfen. Jeder Eingriff in natürliche Gegebenheiten, sei er auf dem Land oder auf dem Wasser, wird von Tieren als potenzielle Veränderung ${ }^{90}$ wahrgenommen. Aufgrund solcher umwelttechnisch beachtenswerten Faktoren sind im Vorfeld solcher Bauten u.a. neben Vereinbarungen mit dem Direktor des Meeresamtes auch ein Bericht über die Prüfung

${ }^{80}$ Ein mit $720 \mathrm{MW}$ geplanter, knapp $23 \mathrm{~km}$ vom polnischen Ort Łeba entfernter OffshoreWindpark.

81 Im Normalfall maximal 8 Jahre (+ 2 Ergänzungsjahre).

82 D. Sołtysiak, Perspektiven zur Entwicklung und Förderung der Offshore - Windparks in Polen, Heiligendamm 2019, S. 7.

83 Ibidem.

${ }^{84}$ M. Krzysztofik, Rechtliche Rahmenbedingungen für Windkraftanlagen in Polen, Berlin 2014, S. 9: Offshore-WEA gehören zur Gruppe I, die von einer Genehmigungspflicht betroffen sind.

85 Ibidem: Die genauen Festlegungen werden für jeden Einzelfall individuell angesetzt.

${ }^{86}$ Homepage der NABU - Natur\&Landschaft - Meere - Offshore-Windparks - Detloff, $Z u$ viel Lärm im Meer.

87 Ibidem.

88 Eine etwa $46 \mathrm{~km}$ von der belgischen Küste entfernte Windparkanlage, die mit 56 Windrädern ausgestattet ist und eine installierte Leistung von $171 \mathrm{MW}$ hervorbringt.

89 Wissenschaftliche Dienste des Deutschen Bundestags, Sachstand: Zu ökologischen Auswirkungen von Windkraftanlagen, Berlin 2019, S. 9.

90 In derselben Untersuchung wurde u.a. festgestellt, dass auch gewisse Gänsearten gezielt ihre Flugrouten verändert haben, um den Offshore-Park nicht zu passieren. 
der Umweltverträglichkeit zu erbringen; das Untersuchungsverfahren der Meeresfauna und der notwendige Umweltschutzbescheid wurden im Battyk Środkowy III-Projekt jeweils zwischen den Jahren 2012 und 2016 eingebracht. ${ }^{91}$ Derartige intensive und zudem langwierige Prozedere können zwar diverse Bauvorhaben im Ostseegebiet zurückwerfen und Investoren, trotz der nun zugesicherten Absicherung, von hohen Finanzierungen abbringen, doch sollte der Tierschutzgedanke und der Schutz der Flora immer als höchstes Gut im Blick bleiben.

\section{Atomkraftwerk in Żarnowiec}

Ein zweites markantes Beispiel entstammt der Atomkraft: Unglücke wie Tschernobyl und Fukushima, die neben umwelt- auch gesundheitsschädigenden Auswirkungen hatten, ${ }^{92}$ sie ließen vor allem in den 2010er Jahren Diskussionen aufkommen, ob die Atomkraft überhaupt noch Potenzial für die Zukunft aufweist oder nicht. ${ }^{93}$ Das Umdenken bezüglich dieser Energiegewinnung ist seit jeher, in Ländern wie Deutschland und der Schweiz, auf gesellschaftlicher wie auch auf politischer Ebene spürbar. ${ }^{94}$ Moderne Ansätze im Bereich der Kernenergie geben aber auch den Ausblick darauf, dass Atomenergie für zukünftige Energieproblematiken in Betracht kommt. Das Generation IV International Forum, ein Forschungsverbund, der v.a. im Bereich der Entwicklung moderner Kernkraftwerke tätig ist, ${ }^{95}$ fertigte etliche Konzepte futuristischer Atomkraftwerke an, die eine Art Renaissance der Atomkraft bedeuten könnten. ${ }^{96}$ So gab es insgesamt sechs Konzepte, wie z.B. den Salzschmelzen-Reaktor oder heliumgekühlte Hochtemperaturreaktoren..$^{97}$ Alle Konzepte sind unter dem Deckmantel konzipiert worden, sicherer, ertragreicher und nachhaltiger, Energie zu generieren. ${ }^{98}$ Doch sind diese Ideen bislang nur auf dem Papier vorhanden und noch nicht in umgesetzter Planung vorzufinden. Polen wäre für die Umsetzung solcher Ideen - planungsrechtlich - prädestiniert, da aufgrund ihrer Energiewende der Weg zu modernen Konzeptionen geebnet wurde. Die bereits oben angesprochene Energiestrategie

91 D. Sołtysiak, op. cit., S. 5.

92 S. Pflugbeil et al., Gesundheitliche Folgen von Tschernobyl - 20 Jahre nach der Reaktorkatastrophe, Berlin 2006, S. 4; C. Pistner, Fukushima - Unfallablauf und wesentliche Ursachen (Teil 1/3), „Fachbeiträge Risikotechnologien“ 9, 2013, S. 413 ff.

93 M. Miersch, M. Bülow, 70 Argumente gegen Atomenergie - Die Zukunft gehört den Erneuerbaren Energien, Berlin 2010, S. 10 ff.; L. Mez, Die Zukunft der Atomkraft nach Fukushima - Warum eine „Renaissance der Atomenergie“ ausbleiben wird, Bonn 2011, S. 10.

94 L. Mez, op. cit., S. 10 f.

95 Die Treffen dieses Forums begannen bereits im Jahre 2000: Homepage des The Generation IV International Forum - About - Origins (abgerufen: 27.03.2021).

96 T. Schulenberg, Ausgewählte Kernreaktoren der Generation IV, Dresden 2011, S. 2 f.

${ }^{97}$ Ibidem, S. 3.

98 Ibidem, S. 2. 
Polityka Energetyczna Polski (PEP2040) ${ }^{99}$ beinhaltet nämlich neben OffshoreWindparks bis 2030, auch die Inbetriebnahme des ersten Atomkraftwerks in Polen im Jahre 2033. ${ }^{100}$ Das übergreifende Ziel im Rahmen der Energiepolitik ist die Sicherstellung eines entsprechenden Niveaus zur Deckung des Energiebedarfs der Wirtschaft und der Bevölkerung, zu konkurrenzfähigen Preisen und auf eine mit den Anforderungen der Umwelt übereinstimmenden Weise. ${ }^{101}$ Das gesamte Nuklearprogramm der polnischen Energiestrategie sieht weiterhin vor, dass weitere Kernkrafteinheiten nach und nach, ca. alle zwei bis drei Jahre, an geeigneten Standpunkten errichtet werden. ${ }^{102}$ Sechs Kernkraftwerke sollen bis 2040 in Polen entstehen. ${ }^{103}$ Da dieses Projekt augenscheinlich noch nicht umgesetzt wurde und planungsrechtliche Voraussetzungen nicht in abgeschlossener Form vorhanden sind, sind an dieser Stelle v.a. die umweltspezifischen Ankerpunkte relevant. Das PEP2040 wurde in Gänze im Rahmen einer nationalen, strategischen Umweltverträglichkeitsprüfung initiiert. ${ }^{104}$ Einer der Gründe für die Entscheidung, im Zuge dieser Strategie u.a. Kernkraftwerke zu errichten, ist das ambitionierte Ziel, den Kohleverbrauch drastisch zu reduzieren und dadurch die $\mathrm{CO}_{2}-\mathrm{Bilanz}^{105} \mathrm{zu}$ verbessern und von Importen aus dem Ausland unabhängiger zu sein. ${ }^{106} \mathrm{Im}$ Jahre 2018 belief sich der Anteil regenerativer Energien noch auf rund 11\%, wobei deren Wert bis 2023 auf 21\% gesteigert werden soll. ${ }^{107}$

\section{Infrastrukturprojekte}

Als weiterer großer Bereich ist die Infrastruktur zu nennen. Polen ist aufgrund seiner zentralen Lage auch auf supranationaler Ebene ein wichtiger Wirtschaftsstandort. So verwundert es nicht, dass das Projekt Autostrada Al durch Polen führt. Bei dieser Autobahn handelt es sich um das wichtigste Autobahnprojekt

${ }^{99}$ In dieser Strategie wurden u.a. vom polnischen Ministerium für Klima und Umwelt drei Säulen festgelegt: 1) Faire Energiewende, 2) Emissionsfreies Energiesystem und 3) Gute Luftqualität.

100 Die Leistung des Kernkraftwerkes wird sich auf etwa 1-1,6 GW belaufen.

101 Österreichisches Umweltbundesamt, Programm für die Polnische Kernenergie, Wien 2011, S. 1.

102 Energieministerium Warschau, Zusammenfassung und Schlussfolgerungen der Umweltprüfung der polnischen Energiepolitik bis zum Jahr 2040.

103 T. Weber, Polnisches Hybridkonzept: Offshore-Windparks nur mit AKW, Website der Erneuerbare Energien 2020 (abgerufen: 27.03.2021).

104 Energieministerium Warschau, Zusammenfassung und Schlussfolgerungen der Umweltprüfung der polnischen Energiepolitik bis zum Jahr 2040.

105 Die Klima- und Energiepolitik der EU hat diese Ideen u.a. vorangetrieben. Diese sieht nämlich seit 2014 vor, eine 40\%ige Reduzierung der Treibhausgasemissionen gegenüber $1990 \mathrm{zu}$ erreichen. Diese Aspekte flossen in das PEP2040 mit ein.

106 Polski Atom, Uchwała w sprawie Polityki energetycznej Polski do 2040 r., https://www. gov.pl/web/polski-atom/uchwala-w-sprawie-polityki-energetycznej-polski-do-2040-r. (abgerufen: 27.03.2021).

107 GTAI, Fact Sheet - Polen - Nachbar mit großem Potenzial.

Prawo 333, 2021

(C) for this edition by CNS 
Europas. Die Autostrada Al ist eine etwa $565 \mathrm{~km}$ lange polnische Autobahn, die von der an der Ostsee befindlichen Stadt Danzig (Gdańsk) nach Klein Gorschütz (Gorzyczki) in Schlesien führt und in gänzlich ausgebauter Erscheinung, Polen mit Tschechien, der Slowakei und Südeuropa verbindet. ${ }^{108}$ Ein solcher gebietsübergreifender Bau birgt die Notwendigkeit, eine allumfassende, über die Woiwodschaften hinausragende, Planung durchzuführen. Für solche Angelegenheiten ist in Polen - auf gesamtstaatlicher Ebene - das Ministerium für Regionalentwicklung zuständig, welches im Rahmen einer nationalen Raumbewirtschaftungskonzeption ${ }^{109}$ tätig wird. ${ }^{110}$ Diese Raumordnungskonzeption betrifft in erster Linie Investitionsvorhaben, die v.a. überregionaler Natur sind, so z.B. Autobahnen. ${ }^{111}$ Die Autobahn ist Teil des paneuropäischen Verkehrskorridors VI und der Europastraße 75. ${ }^{12}$ Die Ergänzung findet sich durch die deutsche Autobahn A4. ${ }^{113}$

$\mathrm{Zu}$ nennen ist darüber hinaus auch das Zugstreckennetz. Dieses hat in Polen traditionell einen hohen Stellenwert und wird derzeit ausgebaut und modernisiert. ${ }^{114}$ Dabei gibt es erneut Berührungspunkte mit anderen Ländern, wie exemplarisch bei der gemeinsamen Zugstreckenmodernisierung zwischen Angermünde (Deutschland) und Stettin (Szczecin) (Polen). Für dieses sind auf polnischer Seite die Woiwodschaften zuständig. ${ }^{115}$ Auf deutscher Seite ist, nachdem es sich um einen Bundesschienenweg handelt, der Bund zuständig. Dabei ist der polnische Netzausbau auch für die transeuropäische Verbindung relevant. ${ }^{116}$ So sollen im Rahmen des Rail Baltica Projekts die Zugverbindungen für Hochgeschwindigkeitszüge tauglich gemacht werden. ${ }^{117}$ Weiterhin werden einige Züge mit einem einheitlichen europäischen Zugbeeinflussungssystem ETCS (European Train Control System) ausgestattet, was die Zuverlässigkeit und Kapazität im grenzüberschreitenden Bahnbetrieb steigert und gleichsam den durchgehenden europäischen Zugverkehr erleichtert. ${ }^{118}$ Der diesbezügliche Planfeststellungsbeschluss ist im Jahr 2021 geplant. ${ }^{119}$

108 A. Beyer, Faszinierende Projekte Autostrada Al Polen, Hamm 2013.

109 Dieses ist - in letzter Konsequenz - vom Sejm, dem polnischen Parlament, zu beschließen.

110 S. Ebert, A. Tölle, M. Wdowicka, op. cit., S. 5, 12.

111 P. Rataj, op. cit., S. 10.

112 Polen (eautobahn.eu) (abgerufen: 8.09.2021)

$113 \mathrm{https} / / /$ de.wikivoyage.org/wiki/Autostrada_A1 (abgerufen: 27.03.2021).

114 Homepage der GTAI - Trade - Branchen - Branchenbericht - Polen - Polen investiert weiter in die Bahninfrastruktur (abgerufen: 27.03.2021); Verkehrsrundschau, Polnische Eisenbahn - Infrastruktur wird moderner, 2015 (abgerufen: 27.03.2021).

115 Homepage „Europa Sachsen-Anhalt“ - Internationales - Partnerregionen des Landes Wojewodschaft Masowien - Stellung der Wojewodschaften im polnischen Staatsaufbau (abgerufen: 27.03.2021).

116 Homepage der European Commission - Transport - Transport Themes - Infrastructure and Investment - Trans-European Transport Network (TEN-T) (abgerufen: 27.03.2021).

117 Homepage der GTAI...

118 Homepage der Bauprojekte der Deutschen Bahn AG - Bauprojekte - Angermünde - Grenze D/PL, S. 2, 2021 (abgerufen: 27.03.2021).

119 Ibidem. 
Auch bezüglich des Luftverkehrs werden derzeit große Anstrengungen unternommen. Zur Entlastung der bereits vorhandenen Flughäfen soll ein neuer Zentralflughafen entstehen. ${ }^{120}$ Dabei handelt es sich um ein Projekt, welches unter die direkte Zuständigkeit der Regierung fällt. In Deutschland fallen ähnliche Großprojekte aufgrund der spezialgesetzlichen Regelungen in die Zuständigkeit der betroffenen Bundesländer. ${ }^{121}$

Zusammenfassend lässt sich feststellen, dass sich beide Länder ihrer Verantwortung bezüglich des reibungslosen Güter- und Personenverkehres sowohl im eigenen Land als auch über die Landesgrenzen hinaus bewusst sind und entsprechend zukunftsträchtige Investitionen vornehmen.

\section{Zusammenfassung und Ausblick}

Zusammenfassend bietet sich neben der Darstellung der Unterschiede und Gemeinsamkeiten auch ein Ausblick an. Deutschland und Polen scheinen im ersten Moment sehr unterschiedlich zu sein. Betrachtet man die beiden Länder jedoch genauer, sind gewisse Gemeinsamkeiten nicht zu übersehen.

$\mathrm{Zu}$ nennen ist zunächst der dreigliedrige Staatsaufbau - ungeachtet der unterschiedlichen föderalistischen Konstruktion. ${ }^{122}$ Ebenso bestehen unterschiedliche Verfahren und Normen, welche Fachplanungen, Gesamtplanungen für den gesamten Staatsraum und Planungen in einzelnen Teilbereichen eröffnen. ${ }^{123}$ Dementsprechend sind die Vereinbarungen zwischen Deutschland und Polen auf unterschiedlichen Ebenen angesiedelt. ${ }^{124}$

Die Raumplanung in Deutschland orientiert sich v.a. am Gegenstromprinzip, wohingegen die Raumwirtschaft in Polen nach dem Prinzip der Anpassung und Konsultation stattfindet; gleichwohl sind beide in der Methodik vergleichbar. ${ }^{125}$ Der polnische Begriff der „Raumwirtschaft“ macht jedoch deutlich, dass auf polnischer Seite die Wirtschaftlichkeit und die finanziellen Komponenten planungsrechtlicher Projekte im Vordergrund stehen. ${ }^{126}$ In Deutschland wiederum ist

120 Homepage der GTAI...

121 Homepage des Landes Berlin - Politik, Verwaltung, Bürger - Senatsverwaltungen - Senatsverwaltung für Umwelt, Verkehr und Klimaschutz - Verkehr - Verkehrsplanung - Luftfahrt Flugplätze - Flughafen Berlin-Brandenburg (BER) (abgerufen: 27.03.2021).

122 S. Ebert, A. Tölle, M. Wdowicka, op. cit., S. 11.

123 Ibidem, S. 3 ff.

124 Exemplarisch das „Abkommen über die Zusammenarbeit im grenznahen Raum“ vom 22.11.1992 zwischen den drei Grenzbundesländern und den Grenzwojewodschaften, vgl. W. Okon, Raumordnung und Landesplanung in der deutsch-polnischen Region Odermündung, [in:] Strukturwandel in Ostdeutschland..., S. 176.

125 S. Ebert, A. Tölle, M. Wdowicka, op. cit., S. 5.

126 Ibidem, S. 3. 
die Schaffung vergleichbarer Lebensverhältnisse und die Vermeidung räumlicher Zersplitterung, insbesondere durch konträre Nutzungsvorstellungen, ein zentrales Anliegen. Die polnische Nutzung hat insoweit eine gewisse Nähe zu anderen zentralstaatlichen Systemen, wie beispielsweise Frankreich. ${ }^{127}$

Dabei ist zu beachten, dass diese einzelstaatlichen Ideen und Vorstellungen durch die supranationalen Vorgaben, insbesondere durch solche der Europäischen Union, angeglichen werden. Unterschiedlichste Richtlinien und Vorgaben, wie exemplarisch die Fauna-Flora-Habitat-Richtlinie, die SUP-Richtlinie, das Natura-2000-Konzept oder die SUPG-Richtlinie, haben allgemein verbindliche Prüfelemente in den unterschiedlichen Genehmigungsverfahren hervorgebracht. Als mittelbare Effekte können sich beispielsweise auch Vorgaben für die Standortsuche bestimmter raumbedeutsamer Projekte ergeben. ${ }^{128}$

Untersuchungen aus früheren Jahren wurde festgestellt, dass planerische und raumordnerische Konzepte die jeweiligen Entwicklungen stärken, gleichsam jedoch nicht auf die konsequente Stärkung des Grenzraums ausgerichtet sind. ${ }^{129}$ Ein gemeinsames Beschreiten der Zukunft würde dabei mehr Optionen eröffnen, um unter anderem den Vorgaben der Europäischen Union im Bereich der Energiepolitik nachzukommen. Eine stärkere Fokussierung künftiger Investitionen auf die Ostsee und den Grenzraum könnte bisher ungenutztes Potenzial erschließen. Neben dem Stopp von Abwanderungen könnte bei kombiniertem Wissen und gut ausgebauter Infrastruktur, die an einzelnen Standorten gewonnene Energie auf andere Teilbereiche der jeweiligen Länder verteilt werden.

In Polen wie auch in Deutschland sind unterschiedliche Herangehensweisen zu erkennen, um mit den Themen Versiegelungsproblematik, Baudynamik und nachhaltigem Errichten von Infrastrukturen umzugehen. Die Herausforderungen, die in näherer Zukunft in Polen und in Deutschland ähnlicher Natur sein werden, zeichnen schon heute ein erkennbares Gesicht ab. Die Geburtenrate ist in beiden Ländern sinkend. Die Bevölkerung selbst wird durch immer höhere medizinische Standards im Schnitt älter, was letztlich dazu zwingt, hauptsachlich im gemeindlichen Bereich, mehr auf altergerechtes Bauen zu setzen. Die Reaktion ist in beiden Ländern durchaus unterschiedlich. Deutschland fährt ein systematisches und sehr verfahrensintensives planungs- und baurechtliches Konzept, was v.a. bei Groß-

127 Aus historischen Gründen wurde in Frankreich der Siedlungsprozess nicht in umfangreichem Maße gesteuert: Das Grundstück wurde eigentumsrechtlich als unveränderbar angesehen: J.J. Terrin, A few factors in understanding French town planning, „Raumordnung und Raumplanung" 2, 2008, S. 170.

128 P. Rataj, op. cit., S. 35.

129 Ministerium für Infrastruktur und Raumordnung des Landes Brandenburg/Senatsverwaltung für Stadtentwicklung des Landes Berlin/Deutsche Gesellschaft für Technische Zusammenarbeit (GTZ) GmbH, Abschlussbericht: Untersuchungen der grenznahen und grenzüberschreitenden Infrastrukturplanungen im Bereich der deutsch-polnischen Grenze im Wirkungsbereich des Verkehrskorridors der Rail Baltica, S. 3. 
projekten immer wieder auf Kritik und rechtliche Konsequenzen stößt. Klagewellen, die Baustopps und weitere, zeitfressende Überprüfungen bei öffentlichen Projekten nach sich ziehen können, sind in Deutschland keine Seltenheit. Mit dem Kostenfaktor, der in Deutschland (vornehmlich Süddeutschland) durch eine hohe privatwirtschaftliche Bauentwicklung in die Höhe getrieben wird, kann die öffentliche Hand, vor allem kleinere Kommunen und Landkreise, nicht mehr mithalten. Durch so eine Diskrepanz zwischen öffentlicher und privater Hand entstehen Diskontinuitäten, die am Ende das öffentliche Bauwesen ins Abseits schießt.

In Polen wiederum ist eine stabile, wirtschaftliche Baudynamik, sei es auf öffentlichem oder auf privatem Boden, deutlich spürbar. Inländische Fachkräfte, die in den letzten Jahren zumal Projekte im Ausland mitbetreut haben, wurden peu à peu durch ausländische Facharbeiter vornehmlich aus der Ukraine ersetzt. Der Fokus auf die Raumwirtschaft, der anders als in Deutschland verstärkt ökonomische Gesichtspunkte in den Vordergrund stellt, tut im Bereich des Bauwesens sein Übriges. Insgesamt bleibt die Ausgewogenheit von ökonomischer und ökologischer Perspektive in beiden Staaten die große Herausforderung der Raumordnung.

\section{Literaturverzeichnis}

Bachmann K., Zur Entwicklung der polnischen Demokratie, „APuZ - Zeitschrift der Bundeszentrale für politische Bildung" 10-11, 2018.

Battis U., Kersten J., Europäische Raumentwicklung, „EuR” 1, 2009.

Baumann S., Elsner C., de Graaf D., Hoffmann G., Martens K., Noack C., Plehn W., Ries L., Thalheim D., 1987-2017: 30 Jahre Montrealer Protokoll, Dessau-Roßlau 2017.

Beyer A., Faszinierende Projekte Autostrada A1 Polen, Hamm 2013.

Ceglarz A., Die polnische Energiepolitik, Warszawa 2020.

Dörr D., Raumordnung und Landesplanung, [in:] Bes. VwR, hrsg. D. Ehlers, M. Fehling, H. Pünder, Bd. 2, Heidelberg 2020.

Ebert S., Tölle A., Wdowicka M., Planung in Deutschland und Polen aus kommunaler Perspektive - Planowanie w Polsce i w Niemczech z perspektywy gminy, Hannover-Poznań 2012.

Energieministerium Warschau, Zusammenfassung und Schlussfolgerungen der Umweltprüfung der polnischen Energiepolitik bis zum Jahr 2040, Warschau 2021.

Grebing H., Schöllgen G., Willy Brandt, Bonn 2013.

Großmann G.U., Die Welt der Burgen, München 2013.

Isensee J., Idee und Gestalt des Föderalismus im Grundgesetz, [in:] Handbuch des Staatsrechts, Bd. 6, hrsg. P. Kirchhof, Heidelberg 2008.

Krzysztofik M., Rechtliche Rahmenbedingungen für Windkraftanlagen in Polen, Berlin 2014.

Langhagen-Rohrbach C., Raumordnung und Raumplanung, Darmstadt 2010.

Mez L., Die Zukunft der Atomkraft nach Fukushima - Warum eine „Renaissance der Atomenergie“ ausbleiben wird, Bonn 2011.

Miersch M., Bülow M., 70 Argumente gegen Atomenergie - Die Zukunft gehört den Erneuerbaren Energien, Berlin 2010. 
Ministerium für Infrastruktur und Raumordnung des Landes Brandenburg/Senatsverwaltung für Stadtentwicklung des Landes Berlin/Deutsche Gesellschaft für Technische Zusammenarbeit (GTZ) GmbH, Abschlussbericht: Untersuchungen der grenznahen und grenzüberschreitenden Infrastrukturplanungen im Bereich der deutsch-polnischen Grenze im Wirkungsbereich des Verkehrskorridors der Rail Baltica, Potsdam 2007.

Misztal S., Landesplanung in Polen, „Plan: Zeitschrift für Planen, Energie, Kommunalwesen und Umwelttechnik" 6, 1963.

Niewiadomski Z., Turowski G., Deutsch-Polnisches Handbuch der Planungsbegriffe. Polsko-niemiecki leksykon pojęć planistycznych, Hannover-Warszawa 2001.

Okon W., Raumordnung und Landesplanung in der deutsch-polnischen Region Odermündung, [in:] Strukturwandel in Ostdeutschland und Westpolen, hrsg. M. Stroll, Hannover 2004.

Ostojski P., Piątek W., Legalisierung von illegalen baulichen Anlagen in Polen und Deutschland, „Landes- und Kommunalverwaltung” 4, 2014.

Österreichisches Umweltbundesamt, Programm für die Polnische Kernenergie, Wien 2011.

Pflugbeil S., Paulitz H., Claußen A., Schmitz-Feuerhake I., Gesundheitliche Folgen von Tschernobyl - 20 Jahre nach der Reaktorkatastrophe, Berlin 2006.

Pistner C., Fukushima - Unfallablauf und wesentliche Ursachen (Teil 1/3), „Fachbeiträge Risikotechnologien" 9, 2013.

Ptak R., Vom Ordoliberalismus zur Sozialen Marktwirtschaft, Wiesbaden 2004.

Rataj P., Die Grundzüge des polnischen Planungsrechts, [in:] Die Grundzüge des polnischen Planungsrechts, hrsg. Botschaft der Republik Polen in der Bundesrepublik Deutschland, Berlin 2012.

Rohrig K., Richts C., Bofinger S., Jansen M., Siefert M., Pfaffel S., Durstewitz M., Energiewirtschaftliche Bedeutung der Offshore-Windenergie für die Energiewende, Berlin 2013.

Schmuck O., Unser G., Zeitbilder: Die Europäische Union, Bonn 2018.

Schneider J., Die Ursachen für den Zusammenbruch der Sowjetunion und der DDR (1945-1990), Stuttgart 2017.

Schulenberg T., Ausgewählte Kernreaktoren der Generation IV, Dresden 2011.

Schumacher J., Umweltprüfung, [in:] Raumordnungsgesetz, hrsg. K. Werk, J. Albrecht, Wiesbaden 2012.

Schwarzer O., Sozialistische Zentralplanwirtschaft in der SBZ/DDR, Stuttgart 1999.

Selke W., Deutsch-Polnisches Haus: Auf dem Wege zu einer grenzüberschreitenden Wirtschaftsregion in Mitteleuropa?, [in:] Strukturwandel in Ostdeutschland und Westpolen, hrsg. M. Stroll, Hannover 2004.

Slapa S., Pfohl M., Pietschman H., Zemke R., Abstimmungsprozesse in Grenzregionen - Der Umgang mit Ansiedlungen in den Grenzregionen Deutschland-Polen und Deutschland-Tschechien, Berlin 2006.

Sołtysiak D., Perspektiven zur Entwicklung und Förderung der Offshore-Windparks in Polen, Heiligendamm 2019.

Steenblock R., Hartwig I., Die Agenda 2007: Solidarität, Nachhaltigkeit und Innovation, ,integration" 27, 2004.

Steinitz K., Die Wirtschaftsplanung in der DDR - Probleme, Erfahrungen, Schlussfolgerungen, [in:] Erfahrungen und Probleme der Planung und der wirtschaftlichen Entwicklung in der $D D R$, Schlussfolgerungen für die Zukunft, hrsg. Rosa-Luxemburg-Stiftung, Werder an der Havel 2007.

Stüer B., Handbuch des Bau- und Fachplanungsrechts, München 2015.

Terrin J.J., A few factors in understanding French town planning, „Raumordnung und Raumplanung" 2, 2008.

Prawo 333, 2021

(C) for this edition by CNS 
Tölle A., Stadt- und Raumentwicklung Polen, 2018, [in:] Handwörterbuch der Stadt- und Raumentwicklung, hrsg. ARL, Hannover 2018.

Ulrich R., Der Übergang zur Marktwirtschaft in der CSFR, Polen und Ungarn: Ausgangsbedingungen und erste Schritte, Berlin 1991.

Wagner W., EU-illionen für Straßen in Grenzregion zu Polen, „Schweriner Volkszeitung” 2016. Website der Schweriner Volkszeitung - über die Suchleiste „EU-Millionen für Straßen in Grenzregion zu Polen“.

Winter G., Kalichava K., Rechtstransfer und Eigendynamik in Transformationsländern, „ZaöRV” 79, 2017.

Wissenschaftliche Dienste des Deutschen Bundestags, Dokumentation: Aufbau eines föderalistischen Staatswesens, Berlin 2019.

Wissenschaftliche Dienste des Deutscher Bundestags, Sachstand: Zu ökologischen Auswirkungen von Windkraftanlagen, Berlin 2019.

\section{Internetquellen}

Homepage der Bauprojekte der Deutschen Bahn AG, Bauprojekt: Angermünde - Grenze D/PL, 2021, https://bauprojekte.deutschebahn.com/p/angermuende-stettin.

Becker N., Deutschland bleibt Polens wichtigster Handelspartner, GTAI Wirtschaftsumfeld, https:// www.gtai.de/gtai-de/trade/wirtschaftsumfeld/bericht-wirtschaftsumfeld/polen/deutschlandbleibt-polens-wichtigster-handelspartner-102436.

Bundesministerium für Wirtschaft und Energie, Windklimatologie, https://www.erneuerbare-energien.de/EE/Redaktion/DE/Standardartikel/Offshore-Windenergie/forschung-windklimatologie.html.

Bundesstiftung Aufarbeitung, Friszke A., Widerstand und Opposition im Kommunismus 1945-91, 2016, https://www.dissidenten.eu/fileadmin/user_upload/Polen/Oppositionsgeschichte/dissidenten_eu/Polnische-Oppositionsgeschichte.pdf.

Bundesministerium für Verkehr, Bau und Stadtentwicklung, Raumordnerische Zusammenarbeit in deutsch-polnischen Grenzraum, 2012, https://www.bbsr.bund.de/BBSR/DE/veroeffentlichungen/ministerien/bmvbs/sonderveroeffentlichungen/2012/DeutschPolnischerGrenzraum.html.

Detloff C. (NABU), Zu viel Lärm im Meer, https://www.nabu.de/natur-und-landschaft/meere/offshore-windparks/index.html.

eAUTOBAHN, Meldungen aus Polen, http://www.eautobahn.eu/html/polen.html.

EU-Info, Gesetzgebung, https://www.eu-info.de/europa/eu-richtlinien-verordnungen.

Homepage „Europa Sachsen-Anhalt“, Stellung der Wojewodschaften im polnischen Staatsaufbau, https://europa.sachsen-anhalt.de/internationales/partnerregionen-des-landes/wojewodschaftmasowien/stellung-der-wojewodschaften-im-polnischen-staatsaufbau/.

Europäische Kommission, Ein europäisches Naturschutznetz - Chancen oder totaler Stillstand in der Gemeinde?, https://ec.europa.eu/environment/nature/legislation/fitness_check/evidence gathering/docs/Member\%20State\%20Stakeholders/Non-Govermental\%20Organisations/LU/ MS\%20-\%20LU\%20-\%20NGO\%20-\%20Natura\%202000.pdf.

Europäische Kommission, Trans-European Transport Network (TEN-T), https://ec.europa.eu/transport/themes/infrastructure/ten-t_de.

The Generation IV International Forum, Origins, https://www.gen-4.org/gif/jcms/c_9334/origins.

GTAI, Polen: Nachbar mit großem Potential, https://www.gtai.de/gtai-de/trade/wirtschaftsumfeld/ fact-sheet/polen/polen-nachbar-mit-grossem-potenzial-207182.

GTAI, Polen investiert weiter in die Bahninfrastruktur, https://www.gtai.de/gtai-de/trade/branchen/ branchenbericht/polen/polen-investiert-weiter-in-die-bahninfrastruktur-144036. 
Homepage des Landes Berlin, Flughafen Berlin-Brandenburg (BER), https:/www.berlin.de/sen/ uvk/verkehr/verkehrsplanung/luftfahrt/flugplaetze/flughafen-berlin-brandenburg-ber/.

MORO-Projekt „Raumbeobachtung Deutschland und angrenzende Regionen“, https://www.sig-gr. eu/de/sig-gr/cooperations/projet_moro_observation_territoriale_allemagne_et_regions_limitrophes.html.

Polski Atom, Uchwata w sprawie „Polityki energetycznej Polski do 2040 r., https://www.gov.pl/ web/polski-atom/uchwala-w-sprawie-polityki-energetycznej-polski-do-2040-r.

Statista, Polen: Bruttoinlandsprodukt von 1980 bis 2019, https://de.statista.com/statistik/daten/studie/14410/umfrage/bruttoinlandsprodukt-bip-in-polen/.

Statista, Rangfolge der wichtigsten Handelspartner Deutschlands nach Wert der Exporte im Jahr 2020, https://de.statista.com/statistik/daten/studie/2876/umfrage/rangfolge-der-wichtigsten-handelspartner-deutschlands-nach-wert-der-exporte/\#: :text=Bezogen\%20auf\%20die\%20Exporte\% 20waren,95\%2C98\%20Milliarden\%20Euro.

Statistisches Bundesamt, 11. Koordinierte Bevölkerungsvorberechnung, Bericht 2006, https://www. destatis.de/DE/Themen/Gesellschaft-Umwelt/Bevoelkerung/Bevoelkerungsvorausberechnung/Publikationen/Downloads-Vorausberechnung/bevoelkerung-deutschland-2050-bericht-5124203069004.pdf? _blob=publicationFile.

Verkehrsrundschau, Polnische Eisenbahn - Infrastruktur wird moderner, 2015, https://www.verkehrsrundschau.de/nachrichten/polnische-eisenbahn-infrastruktur-wird-moderner-1708941.html.

Weber T., Polnisches Hybridkonzept: Offshore-Windparks nur mit AKW, Website der Erneuerbare Energien 2020, https://www.erneuerbareenergien.de/politik/klimapolitik/anti-energiewendewende-polnisches-hybridkonzept-offshore-windparks-nur-mit-akw.

Weber T., Warschau schickt Windkraft in die See, Erneuerbare Energien 2021, https://www.erneuerbareenergien.de/politik/energiepolitik/meereswindkraft-europa-warschau-schickt-windkraftdie-seeVerkehrs.

Wikipedia, Autostrada A 1, https://de.wikipedia.org/wiki/Autostrada_A1_(Polen).

Wikivoyage, Autostrada A1, https://de.wikivoyage.org/wiki/Autostrada_A1.

Zeit, Koalitionsvertrag 2018-2021, https://www.zeit.de/politik/deutschland/2018-02/koalitionsvertrag.pdf?utm_referrer=https $\% 3 \mathrm{~A} \% 2 \mathrm{~F} \% 2 \mathrm{Fwww}$.google.com $\% 2 \mathrm{~F}$.

\section{Spatial Planning in Germany and Poland: A Comparative Legal Sketch}

\section{Summary}

Spatial planning in Germany and Poland has a long history. In addition to individual spatial planning, there are also joint as well as general European approaches to the use of space. The article first shows the legal framework at national and supranational level, followed by an in-depth presentation based on several projects from the energy and infrastructure sectors. Finally, the similarities and differences are worked out.

Keywords: similarities, offshore wind farms, nuclear power station, autostrada A1, central airport. 